\title{
Energetic Particle from CME-Driven Shocks: Spectra, Composition, and Timing
}

\author{
Allan J. Tylka \\ Naval Research Laboratory, Washington DC, USA
}

\begin{abstract}
Very large solar energetic particle (SEP) events occur at the rate of about 10 per year during solar maximum. The primary accelerators in these events are shocks driven by fast coronal mass ejections, in which speeds are greater than about $1000 \mathrm{~km} / \mathrm{s}$. Solar Cycle 23 probably produced the largest fluence of $>10 \mathrm{MeV} /$ nucleon SEPs seen since the start of the Space Age. New instruments on Wind, ACE, SOHO, and other satellites have provided unprecedented detail on the energy spectra, elemental and isotopic composition, ionic charge states, and temporal evolution of these SEP events, as well as their associated CMEs and flares. In this talk, I will review some of the new insights provided by these data. A particular challenge in SEP studies has been the very large event-to-event variability in composition and spectral characteristics, particularly at energies above a few tens of $\mathrm{MeV}$ per nucleon. I will discuss recent efforts to understand this variability in terms of seed populations and shock geometry. I will also review recent studies of the time at which SEPs first appear on the Sun-Earth magnetic field line and the implications of these studies for the conditions under which SEP production was initiated.
\end{abstract}

Keywords. Sun: coronal mass ejections (CMEs), Sun: particle emission

\section{Discussion}

Yousef: 1) You have shown that Fe/O increases with energy, would that imply preferential acceleration of heavy particles at this domain of energy?

2) Do all relativistic nuclei (particularly) the heavy one arrive with the same speed. Please do comment on $\mathrm{Pb}$ observation.

3) Do you also find or find indication of relativistic electron accelerated at the same time with same velocities? Please look at my acceleration paper in this symposium.

TYLKA: 1) No. I would not make that interpretation. Instead, the increase with energy reflects a change in the accessible seed population, coupled with a steepening in the shock-accelerated spectra, as the CME moves outward from the Sun.

2) No, there is velocity dispersion in the particle arrivals. All species have a spectrum of energies. The ultra-heavy ions $(\mathrm{Z}>30)$ have been observed from $100 \mathrm{keV} / \mathrm{nuc}$ to $\sim 10 \mathrm{MeV} /$ nuc. But this is a limitation of presently-available instrumentation. For more on the ultra heavies, see Reames (2000, ApJLett); Reames \& Ng (2004, ApJ); Mason et al. (2004, ApJ).

3) A spectrum of electrons is also produced along with the heavy ions.

KAHLER: Is it not the case that we can assume a simple shock geometry based on the CME longitude, in which a CME near central meridian is associated with a quasiperpendicular shock and a CME around W80 with a quasi-parallel shock?

TYLKA: These high energy SEPs are generally being produced at very low altitude, perhaps just a few solar radii according to the timing studies of Bieber et al. (2004). At these altitudes, the open magnetic field is nominally radial, and the shock geometry will 
be determined by the details of the CME and the ambient medium. Intuition based on the large scale Parker spiral is unlikely to be very helpful at these altitudes.

Kouтснму: What about more "geometric" parameters determining SEP composition/ energy, like center-limb effect and E/W asymmetry of the solar source of SEP?

TYLKA: The gradual events with enhanced Fe/O at high energies and Fe with a harder power-law than O are found from sources all across the Sun. One such event (1999, January 20) had its source region region at E95 according to Cane et at 2001. This behavior contrasts to classic, impulsive events in which ${ }^{3} \mathrm{He} /{ }^{4} \mathrm{He}>10 \%$ at a few $\mathrm{MeV} / \mathrm{nuc}$. In those events, $85 \%$ are found at longitudes between W30 and W80, according to the longitude distribution provided by Reames (1999). The spread in the distribution is primarily determined by variation in solar-wind speed. By comparison only $\sim 40 \%$ the events with enhanced $\mathrm{Fe} / \mathrm{O}$ at $>30 \mathrm{MeV} /$ nuc are found at W30-W80.

GANG Li: In the spectrum show in the bow shock acceleration diagram, does the seed population extend to high energies and the power law is a simple increase of intensity? Or does the seed population is more a power-law with a roll-over, but the acceleration process produces the power law at high energies?

TYLKA: To my recollection, the authors (Meziane et al., JGR 2001) of the bow shock papers did not discuss the spectrum of the incident seed particles. However, given the small size of the bow shock, the slope of the power law may very well reflect the spectra of the seed particles. Never- theless, it is significant that the power-law spectra are only seen at quasi-perpendicular configurations. 